\title{
Right-left response differentiation in rats with unilateral cortical spreading depression*
}

\author{
BARBARA BERGER $†$ and T. P. HOGAN $\dagger \dagger$ \\ McGill University, Montreal, P.Q., Canada
}

Food-deprived rats were trained during unilateral cortical spreading depression (CSD) to make a conditional left-right response differentiation in a $\mathrm{T}$-maze. Interhemispheric transfer, veridical or paradoxical, did not result with the opposite hemisphere depressed. A strong tendency to enter the arm ipsilateral to the depressed hemisphere suggested that CSD may serve as an orienting cue. These results contradict earlier experiments which reported reversal responding during test trials. The discrepancy can be accounted for in terms of incomplete depression and the lateral cue it provided in the earlier studies.

Ray \& Emley (1964, 1965) have studied the interhemispheric transfer of a discrimination task engram by eliciting cortical spreading depression (CSD) unilaterally during acquisition of the discrimination. In their experiments, rats were trained with one hemisphere depressed to make a conditional left-right response differentiation in a T-maze. When tested under the same conditions but with the opposite hemisphere depressed, nearly all the animals responded by running into the arm opposite to the one that was correct during training (Ray \& Emley, 1964, 1965).

These results are of renewed interest in light of the support they provide for the "reversal hypothesis" of interhemispheric memory transfer put forth by Noble $(1966,1968)$ and recently amplified by Corballis \& Beale (1970). In brief, the hypothesis states that a memory trace stored in either hemisphere will undergo reversal along the right-left axis as it is projected across the homotopic connections of the interhemispheric fiber systems.

Although Ray and Emley did not interpret their observations of reversal responding in these terms, the present experiment was designed to repeat their experimental paradigm and to assess its implications, if any, for the reversal hypothesis.

* This research was part of an undergraduate research project at $\mathrm{McGil}$ University and was supported in part by a grant from the National Research Council of Canada to M.C. Corballis. We also acknowledge with gratitude the supervision and assistance of Dr. Michael Corballis.

+Now at the University of Waterloo, Waterloo, Ont., Canada.

++ Requests for reprints should be sent to T. P. Hogan. Department of Psychology, University of Waterloo, Waterloo, Ont. Canada.

\section{SUBJECTS}

Eight naive male rats of the Wistar strain were obtained from the Canadian Breeding Farm and Laboratory at an initial weight of 240-280 g. Ss were put on a 23-h food-deprivation schedule which commenced 5 days prior to pretraining and was maintained throughout the experiment.

\section{SURGERY}

Preparation of Ss for the application of potassium chloride (KCl) to the cortex consisted of drilling bilateral holes between the coronal and lamboidal sutures and suturing the loose skin to a hollow plastic ring which encircled the two holes. The holes were washed out daily and kept free of foreign matter by means of a rubber cap fastened to the ring.

\section{PROCEDURE}

The apparatus was a simple T-maze with a 60-W light bulb suspended $40 \mathrm{~cm}$ above the choice point. Prior to the experimental sessions and before surgery, the animals were allowed to explore the entire maze and were given access to a single food pellet in each arm for $10 \mathrm{~min}$ each day for 3 days.

The discrimination the animals had to learn was that when the light over the maze was on, one arm contained the food pellet, and when the light was off, the other arm was correct. The sequence of correct responses was random, but there were never more than two consecutive correct responses on one side. Half the Ss were trained with the right hemisphere depressed, half with the left. Filter paper soaked in a $25 \%$ solution of $\mathrm{KCl}$ was applied to the dural membrane of the hemisphere to be depressed $5-10 \mathrm{~min}$ before the start of the training trials. A rat was not placed in the startbox unless it exhibited unilateral hypesthesia in the limbs contralateral to the depressed hemisphere, a behavioral sign of unilateral CSD (Carew, Crow, \& Petrinovich, 1970). The same test was made immediately after a session as well. As many training trials were given as could be run within $30 \mathrm{~min}$ each day until Ss achieved the criterion of six consecutive or seven out of eight correct responses.

A single transfer session was given the day following achievement of criterion. The conditions were the same as during training trials, except for two differences: the opposite hemisphere was depressed, and both arms of the maze contained food reward.

\section{RESULTS}

The main findings are presented in Table 1. All Ss reached criterion by the sixth experimental session, except for three animals which were eliminated due to unsuccessful elicitation of CSD. The successful Ss made as many incorrect as correct responses in the transfer trials. This result is deceptively random, since four of the five rats always went to the arm of the maze ipsilateral to the depressed hemisphere and all Ss chose this side on the first trial whether the light was on or off. The same preference for the arm ipsilateral to the depressed hemisphere was shown in the early acquisition trials as well. It should be noted that during transfer trials, Ss were reinforced in whichever arm they entered. This would have the effect of exaggerating the original unbiased preference. With this qualification in mind, the data do not exhibit the reversal responding observed by Ray and Emley.

There appeared to be some retention of the general nature of the task in test trials, since the latencies for the first eight trials of testing were shorter than for the first eight trials of acquisition $(t=4.1, d f=4, p<.02)$. DISCUSSION

Contrary to the findings of Ray \& Emley $(1964,1965)$, the present experiment offered no evidence whatsoever for reversal in lateral responding with opposite hemispheres functional. In fact, the results showed no indication for interhemispheric transfer, reversed or unreversed, of the

Table 1 Results of Transfer Trials

\begin{tabular}{lcc}
\hline & \multicolumn{2}{c}{ Transfer Trials } \\
\cline { 2 - 3 } & $\begin{array}{c}\text { Proportion } \\
\text { Correct } \\
\text { Sesponses }\end{array}$ & $\begin{array}{c}\text { Proportion } \\
\text { Ipsilateral } \\
\text { Responses }\end{array}$ \\
\hline Rat 1 & $13 / 25$ & $25 / 25$ \\
Rat 2 & $12 / 25$ & $9 / 25$ \\
Rat 3 & $12 / 24$ & $24 / 24$ \\
Rat 4 & $13 / 25$ & $25 / 25$ \\
Rat 5 & $4 / 8$ & $8 / 8$ \\
\hline
\end{tabular}


left-right differentiation, since the choice of response during test trials was not at all contingent on the light. That the latencies were shorter in test trials than in the early acquisition trials can be interpreted as a nonspecific learning effect, mediated subcortically.

Ray and Emley make no mention in their studies of a measure of the behavioral indicant of CSD (such as loss of the placing reflex)-an oversight which would have permitted incomplete depression to have gone undetected. It is possible, then, that, rather than totally depressing the function of a hemisphere, the incomplete CSD simply acted as a lateralized stimulus cue. Thus an animal may have learned, for example, to turn towards the depressed hemisphere when the light was on and away from it when the light was off. This explanation would clearly predict left-right reversal when the opposite hemisphere is depressed.
With complete depression, which we suppose to have been achieved in the present experiment, there would be no such reversal responding, since information about the light cue is denied access to the depressed hemisphere during acquisition. In this case, choice of response is determined by which hemisphere is depressed, and not by the light.

While our findings do not irrevocably banish the possibility that reversal responding is the result of reversal in lateral orientation of the memory trace as it is transmitted from a functional to a partially depressed hemisphere, they do suggest a more parsimonious explanation in terms of the stimulus cue provided by CSD. The systematic bias produced by CSD rules out the technique as a research tool in testing left-right response differentiation.

It is not altogether clear why there should be a tendency to choose the arm of the maze ipsilateral to the depressed hemisphere. One possibility is that the visual field contralateral to the depressed hemisphere is functionally blind, so the rat is much more likely to notice the arm on the ipsilateral side.

\section{REFERENCES}

CAREW, T., CROW, T., \& PETRINOVICH, L. Lack of coincidence between neural and behavioral manifestations of cortical spreading depression. Science, 1970, 169 , 1339-1341.

CORBALLIS, M. C.. \& BEALE, I. L. Bilateral symmetry and behavior. Psychological Review, 1970, 77, 451-464.

NOBLE, J. Mirror-images and the forebrain commissures of the monkey. Nature, 1966, 211, 1263-1265.

NOBLE, J. Paradoxical interecular transfer of mirror-image discrimination in the optic chiasm sectioned monkey. Brain Research, $1968,10,127 \cdot 151$.

RAY, O. S., \& EMLEY, G. Time factors in interhemispheric transfer of learning. Science, 1964, 144, 76-78.

RAY, O. S., \& EMLEY, G. Interhemispheric transfer of learning. Life Sciences, 1965, 4, 823-826. 